\title{
Computed tomography coronary angiography: Diagnostic yield and downstream testing
}

\author{
Authors: Saad Fyyaz, ${ }^{\mathrm{A}}$ Jonathan Hudson, ${ }^{\mathrm{B}}$ Olaolu Olabintan, ${ }^{\mathrm{C}}$ Athanasios Katsigris, ${ }^{\mathrm{D}}$ Sarojini David, ${ }^{\mathrm{E}}$ Sven Plein ${ }^{\mathrm{F}}$ \\ and Khaled Alfakih ${ }^{\mathrm{G}}$
}

\section{Background}

The UK National Institute for Health and Care Excellence (NICE) updated its guidelines on stable chest pain in 2016 and recommended computed tomography coronary angiography (CTCA) as first line investigation for all patients with new onset symptoms. We implemented the guideline and audited downstream testing.

Methods

We undertook a retrospective search of the local radiology database from January 2017 to May 2018.

\section{Results}

Six-hundred and fifty-two patients underwent CTCA (mean age of 55 years, 330 were male). Thirty-four patients were found to have severe coronary artery disease (CAD), with 30 undergoing invasive coronary angiography (ICA) which confirmed severe CAD in 22 , a yield of $73 \%$.

Fifty-eight patients were found to have moderate CAD on CTCA with 36 referred for ICA, of which, 33 attended and 18 were found to have severe CAD. Eighteen were referred for imaging stress tests and one was positive. The total yield of severe CAD at ICA was $55 \%$. The majority of patients had normal coronary arteries.

Conclusions

CTCA was an effective rule-out test for most patients. In patients that went on to have ICA, the overall yield of severe CAD was relatively high. This compares well with our previous audit applying the NICE 2010 guidelines which recommended ICA for all high probability patients wherein the yield of severe CAD was $30 \%$.

KEYWORDS: Audit, CT coronary angiography, coronary artery disease, guidelines, NICE

Authors: ${ }^{\text {A }}$ cardiology specialist registrar, Lewisham and Greenwich NHS Trust, Lewisham, UK; ${ }^{B}$ core medical trainee, Lewisham and Greenwich NHS Trust, Lewisham, UK; ${ }^{C}$ core medical trainee, Lewisham and Greenwich NHS Trust, Lewisham, UK;

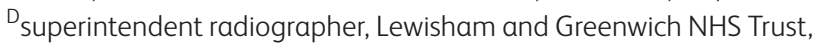
Lewisham, UK; ${ }^{\mathrm{E}}$ consultant radiologist, Lewisham and Greenwich

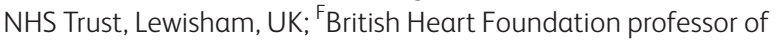
cardiovascular imaging, Leeds Institute of Cardiovascular and Metabolic Medicine, Leeds, UK; ${ }^{G}$ consultant cardiologist, Lewisham and Greenwich NHS Trust, Lewisham, UK

\section{Introduction}

Coronary artery disease (CAD) remains one of the leading causes of death globally. ${ }^{1}$ The diagnosis of significant CAD in patients presenting with stable chest pain is therefore of considerable importance. A number of diagnostic modalities exist to investigate patients presenting with chest pain and suspected CAD, such as imaging stress tests to assess for ischaemia, or coronary angiography to assess for the presence of atherosclerotic coronary stenoses.

Under the 2010 UK National Institute of Health and Care Excellence (NICE) guidelines for the management of stable chest pain, patients were evaluated on the basis of a clinical history and examination, resting 12-lead electrocardiography and a pre-test probability risk score based on typicality of chest pain, cardiac risk factors, age and gender. ${ }^{2}$ Subsequent assessment was based on probability of CAD, with high probability patients recommended to have invasive coronary angiography (ICA), moderate probability patients to have imaging stress tests and low probability patients to have cardiac computed tomography (CT).

We previously implemented the NICE 2010 guidelines locally and referred all patients with high probability of CAD for ICA. We audited this practice and found a low yield of severe CAD of just $30 \%$ in those referred for ICA. ${ }^{3}$ This finding is consistent with the observation in major registries that a risk score led approach overestimates the likelihood of CAD. ${ }^{4}$ There is a general low prevalence of severe CAD at ICA in current cardiology practice, as described in a large American registry, which demonstrated a yield of $38 \%{ }^{5}$ Thus, under the 2010 NICE guidelines, large numbers of patients were exposed to ICA, with a risk of adverse event and relatively low yield of severe CAD.

In response to this issue and the anticipated cost savings, NICE updated the stable chest pain guidelines in 2016 with two significant changes. ${ }^{6}$ The first was in recommending CT coronary angiography (CTCA) as the first-line investigation for all patients with new onset stable chest pain, and the second was in the removal of the risk score.

CTCA is an established test in populations with low-intermediate probability of having CAD because of its excellent negative predictive value and very good sensitivity. ${ }^{7}$ CTCA was equally effective to imaging stress tests in the PROMISE trial, which randomised 10,000 chest pain patients with low-intermediate probability of CAD, to either CTCA or imaging stress tests, and found no difference in outcome. ${ }^{8}$ However, CTCA has a lower positive predictive value, and can overestimate the severity of a stenosis, particularly where there is marked coronary calcification. ${ }^{9}$ 
Currently, there is very limited evidence base for the use of CTCA in patients with higher likelihood of CAD. ${ }^{10,11}$ Thus, the expanded use of CTCA to all patients with stable chest pain in the updated NICE guidelines was met with some criticism. ${ }^{10}$

Since 2017, we have locally adopted the NICE 2016 guidelines and have audited downstream testing following CTCA to assess rates of subsequent imaging stress tests, yield of severe CAD at ICA and revascularisation, alongside other incidental findings found on CTCA and their subsequent investigations. We hypothesised that greater use of CTCA in higher likelihood populations may result in high rates of ICA and second-line investigations in this population.

\section{Methods}

All patients referred for CTCA for stable chest pain at our hospital in a 17-month period from January 2017 to May 2018, were identified with a retrospective search of a radiology database.

All CTCA were performed with a 64 slice LightSpeed VCT XTe (GE Healthcare, Waukesha, USA) scanner, using the commercially available protocol (SnapShot Pulse, GE Healthcare, Waukesha, USA) and the following scanning parameters: slice acquisition $64 \times 0.625 \mathrm{~mm}$, SFOV cardiac, z-axis detector coverage $40 \mathrm{~mm}$, gantry rotation time of $350 \mathrm{~ms}$. For adapted tube voltage, 100 $\mathrm{kV}$ was used for small patients and $120 \mathrm{kV}$ for all other patients. Prospective gating was the standard acquisition protocol. CTCA images were reconstructed with slice thickness of $0.625 \mathrm{~mm}$, on an external workstation (ADW 4.5, GE Healthcare, Waukesha, USA). Patients were beta-blocked aiming to achieve a heart rate of $<60$ beats per minute; $80 \mathrm{~mL}$ of ivoersol (Optiray $350 \mathrm{mg}$ Iodine $/ \mathrm{mL}$, Guerbet, Villepinte, France), at a flow rate of $6 \mathrm{~mL} / \mathrm{s}$, was injected into an antecubital vein. Bolus tracking was used with a region of interest placed into the ascending aorta. CTCA were reported by a cardiologist or a radiologist, with the radiologist reporting on all extra-cardiac findings.

The department's recommendation is for patients found to have less than $50 \%$ coronary artery stenosis to be managed medically, patients with $50-70 \%$ stenosis to be referred for stress echocardiography and those with a coronary artery stenosis $>70 \%$ or $>50 \%$ stenosis of the left main stem (LMS) to proceed to ICA.

Data regarding second-line investigations and revascularisation were recorded retrospectively from the hospitals' electronic patient records. Recommendations for incidental findings were within the CTCA report and in line with British Thoracic Society nodule guidelines. The ICAs were performed in the standard way and reported by experienced interventional cardiologists.

\section{Results}

A total of 749 patients were referred for CTCA between 01 January 2017 and 31 May 2018. A total of 97 patients did not attend for CTCA. Hence, a total of 652 patients underwent CTCA, of which 330 were male and 322 were female, with an average age of 55 years \pm 11 years. (Table 1 ).

\section{Severe disease ( $>70 \%$ stenosis of any epicardial} vessel / >50\% LMS stenosis)

A total of 34 patients were reported to have severe CAD stenosis by CTCA (Fig 1). Thirty-three of these patients were referred for
Table 1. Patient demographics, degree of coronary

stenosis on computed tomography coronary

angiography and the European Society of

Cardiology risk score

\begin{tabular}{|c|c|}
\hline Total patients, $n$ & 652 \\
\hline Male, $\mathrm{n}$ & 330 \\
\hline Female, $\mathrm{n}$ & 322 \\
\hline Average age, years & 55 \\
\hline \multicolumn{2}{|l|}{ CTCA stenosis severity: } \\
\hline Severe $(>70 \%), n$ & 34 \\
\hline Moderate $(50-70 \%), n$ & 58 \\
\hline Mild $(30-50 \%), n$ & 62 \\
\hline Normal $(<30 \%)$, n & 462 \\
\hline Inconclusive, $\mathrm{n}$ & 36 \\
\hline \multicolumn{2}{|l|}{ ESC risk score: } \\
\hline High $(>85 \%), n$ & 2 \\
\hline Intermediate-high ( $51-85 \%$ ), n & 149 \\
\hline Low-intermediate (15-50\%), n & 427 \\
\hline Low $(<15 \%), n$ & 70 \\
\hline Indeterminate, $\mathrm{n}$ & 4 \\
\hline
\end{tabular}

ICA, of which two were lost to follow-up, and one patient declined ICA. Of 30 patients undergoing ICA, 22 were confirmed to have severe CAD by ICA ( $73 \%$ yield). The rest were deemed to have moderate CAD only and had medical management.

Of the 22 patients with severe CAD confirmed by ICA, 18 underwent revascularisation; 15 patients by percutaneous coronary intervention (PCI), and three by coronary artery bypass grafting (CABG). From the remaining four patients, two were referred for dobutamine stress echocardiography (DSE) with no inducible ischaemia found in either, and two were managed medically in the first instance. Only one patient directly underwent DSE following CTCA, and no inducible ischaemia was noted.

\section{Moderate disease $(50-70 \%$ stenosis of any epicardial vessel)}

A total of 58 patients were reported to have moderate CAD on CTCA (Fig 1). This generated 55 downstream tests, 36 directly for ICA, 18 for DSE, and one for myocardial perfusion scintigraphy. A further two patients have no further downstream tests planned; one was diagnosed with an advanced lung carcinoma, the other was managed medically. One patient did not attend for follow-up. From the 36 patients who were referred for ICA, only 33 attended; 18 were found to have severe CAD (55\% yield) and 16 patients were revascularised, 10 by PCI and six by CABG. Only 17 of the 18 patients referred for DSE underwent testing after one patient did not attend; only one patient had a positive test and was subsequently revascularised with PCI. 


\section{Mild disease (30-50\% stenosis of any epicardial vessel)}

A total of 62 patients were reported to have mild CAD. Two of these patients were still referred for ICA in context of having ongoing typical symptoms. One patient was found to have severe disease and subsequently revascularised. Ten patients were referred for DSE; two positive studies were referred onwards for ICA, but no significant stenosis was observed. The remaining eight studies were negative and patients were managed medically.

\section{Normal (normal / $<30 \%$ stenosis of any epicardial vessel)}

A total of 462 patients were deemed to have coronary arteries on CTCA. Two patients underwent stress echocardiography, one for anomalous right coronary artery and one to investigate for microvascular angina and both were negative.

\section{Inconclusive studies}

There were a total of 36 inconclusive CTCA studies. Reasons ranged from difficulties with breath holding, elevated heart rates despite the administration of a beta-blocker, contraindication to beta-blockers and, in one case, contrast extravasation. Eight patients were referred for ICA and three patients were revascularised. Four patients underwent repeat CTCA on a higher specification CT scanner at a neighbouring hospital and shown to have normal coronary arteries. Seventeen patients were referred for DSE and two patients were referred for stress perfusion cardiac magnetic resonance imaging.

\section{Overall use of downstream tests and yield of severe CAD at ICA}

The total number of second-line investigations following CTCA were 76 ICAs, 49 imaging stress tests and four repeat CTCAs. This translates to $12 \%$ of the total patient cohort undergoing ICA following CTCA. The yield of severe CAD at ICA following a severe stenosis on CTCA was $73 \%$. The yield of severe CAD at ICA following a moderate stenosis on CTCA was $55 \%$. The overall yield of severe CAD for patients referred directly to ICA following an interpretable CTCA was 63\% (41/65).

\section{Incidental findings}

A significant number of incidental findings were identified and acted upon. Twenty-six per cent (170) of CTCAs demonstrated a total of 202 incidental findings. Of those, 95 (47\%) were thoracic, $58(29 \%)$ were gastrointestinal, 44 (22\%) were cardiac, and five $(2 \%)$ arose from the breast. The two most frequent of the incidental findings were lung nodules (16\%) and hepatic cysts (15\%). A lung malignancy was an incidental finding on two of the scans.

Of the 652 scans performed, $17 \%$ required a downstream investigation to be performed for an incidental finding. The most common downstream investigations required were abdominal ultrasound (40), CT of the thorax (21) and echocardiography (12). Referral to a specialist clinic for further assessment was required in 31 patients including five to breast clinic. The total additional cost of investigations or onwards referral was $£ 12,464$ and on average per patient for the cohort was $£ 19$.

\section{European Society of Cardiology risk score}

The European Society of Cardiology (ESC) guidelines utilise a risk score (RS) to guide further investigation, and deem that the highest $(>85 \%)$ and lowest $(<15 \%)$ need not undergo further investigation as it would be safe to assume they have obstructive CAD or have no obstructive CAD, respectively.

ESC RSs were calculated retrospectively and did not guide the diagnostic pathway. In our cohort, only two patients had an ESC RS > 85\%; one had a moderate stenosis and one had a mild stenosis on CTCA. There were 149 patients with an ESC RS $50-85 \%$; 17 (11\%) of these patients were found to have a severe CTCA stenosis and $23(15 \%)$ to have a moderate stenosis. A further 427 patients had an ESC RS 15-50\%; 17 (4\%) had a severe CTCA stenosis and $32(8 \%)$ had a moderate stenosis. Lastly, 70 patients had an ESC RS $<15 \%$ and two $(3 \%)$ were found to have a moderate stenosis on CTCA.

\section{Discussion}

In our previous published audit, after the implementation of the 2010 NICE guidelines for stable chest pain, the yield of severe CAD at ICA for patients who were referred directly to ICA based on high likelihood of having CAD was $30 \%{ }^{3}$. Following our implementation of the 2016 update of the NICE guidelines, CTCA was used as a first-line test for most patients with new stable chest pain and the overall yield of severe CAD at ICA was now $63 \%$.

Current ESC and American societies of cardiology guidelines still recommend imaging stress tests in patients with high likelihood of CAD, in the absence of evidence supporting the use of CTCA in high-likelihood populations. The NICE 2016 guidelines consider the fact that the majority of patients presenting with stable chest pain to rapid access chest pain clinics (RACPC) are likely to be of low-intermediate likelihood of having CAD. The majority (71\%) of our cohort who underwent CTCA were found to have normal or minimal CAD.

A relatively small number of patients were found to have mild CAD (30-50\% stenosis) and those were mostly managed medically with secondary prevention. Even smaller numbers of patients were found to have moderate CAD (50-70\% stenosis). Sixty-four per cent of those were referred directly for ICA and 33\% underwent imaging stress tests as a gatekeeper to ICA. The choice of secondline investigation in patients with a moderate CTCA stenosis is not consistent and this reflects a lack of clear evidence base or guidelines on how best to manage these patients. The choice of test was therefore clinician guided after consideration of the typicality of the symptoms. Although this approach led to a yield of severe CAD at ICA of $55 \%$, we hypothesise that this could be improved upon further with a higher utilisation of imaging stress tests in this group. The yield of severe CAD at ICA in the small number of patients with a severe stenosis on CTCA was good at $73 \%$.

Our use of imaging stress tests as a gatekeeper to ICA after CTCA is in keeping with NICE recommendations. NICE recognised a potential for higher rates of ICA following CTCA, and in a separate guideline recommended the use of $\mathrm{CT}$ fractional flow reserve (FFR). ${ }^{12}$ CT-FFR is a non-invasive test, which calculates FFR using the CTCA data and computational fluid dynamics, and correlates reasonably well with the invasive FFR, used in the catheter lab, to assess the significance of coronary stenoses. Due to reasons of cost, this has had restricted utility in clinical practice at the time of the audit, and a previous survey of UK cardiologists 


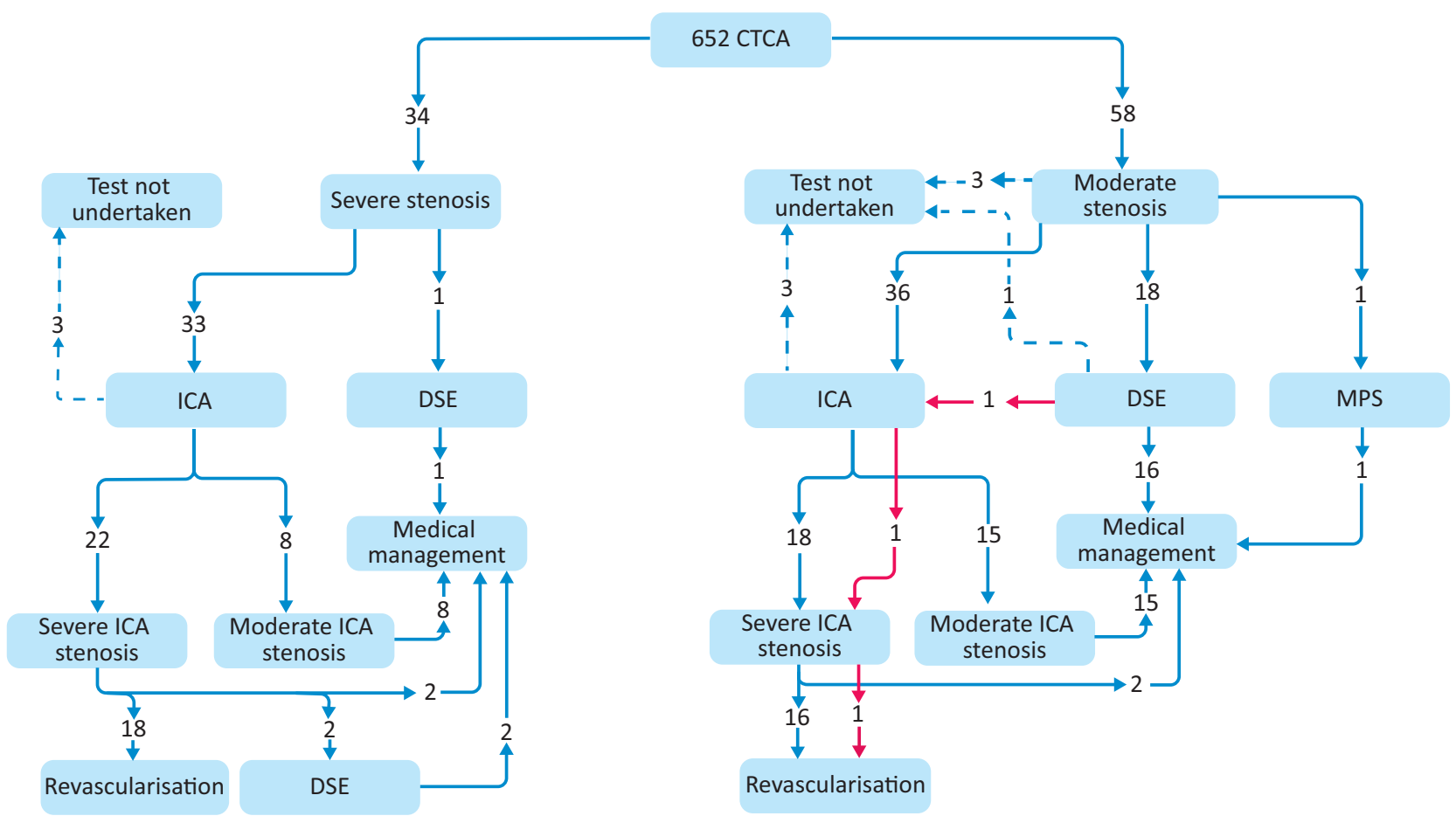

Fig 1. Downstream investigations following computed tomography coronary angiography moderate or severe stenosis. CTCA $=$ computed tomography coronary angiography; DSE = dobutamine stress echocardiography; ICA = invasive coronary angiography; MPS = myocardial perfusion scintigraphy.

suggested that CT-FFR is rarely used. ${ }^{13}$ Importantly, our yield of severe CAD at ICA was better than the published data from a large American registry where the yield of severe CAD was $37.6 \% .^{5}$

There is a concern regarding higher rates of ICA and revascularisation following CTCA in relatively low-risk populations without any difference in mortality. 8,14 The SCOT-Heart trial randomised 4,146 patients with stable chest pain to exercise electrocardiogram (ECG; now obsolete based on NICE guidelines because of its poor accuracy) or exercise ECG plus CTCA. They demonstrated that the combination of CTCA plus exercise ECG had a mortality benefit over the exercise ECG alone. ${ }^{15}$ It remains to be seen if the use of superior imaging stress tests, such as DSE or CTFFR, in patients found to have a moderate stenosis on CTCA may temper the higher rates of ICA and improve outcomes further.

The ESC RS shows the majority of the cohort had a low risk with $76 \%$ of patients having a score $<50 \%$. The ESC RS was only modestly predictive of CAD with a higher incidence of severe stenosis in patients with ESC RS of $50-85 \%$ compared with those with ESC RS of $15-50 \%$ ( $11 \%$ vs $4 \%$, respectively). Although the ESC RS is a validated and updated version of the DiamondForrester model, in a contemporary population it is modelled on patients referred for ICA which differs from RACPC populations. ${ }^{16}$

The expansion of CTCA, as demonstrated by this study, generates a large number of incidental findings both cardiac and extra-cardiac in nature. ${ }^{17}$ The most common incidental findings in our study were hepatic cysts and pulmonary nodules, both requiring downstream scans, and, for the lung nodules, referrals to chest medicine clinics. With the increased use of CTCA, services will need to be able to offer robust pathways for onward referral of incidental findings. It has been suggested in a large Australian study that this can be mitigated by limiting the scanning field of view on CTCA to scan just the heart, if the scanner permits. ${ }^{17}$ This reduces the number of incidental nodules being identified and thereby the associated costs of further investigation / surveillance imaging, and the associated further radiation exposure. Such practice is somewhat controversial with opposing views given the ethical considerations. On one hand, this may prevent or delay diagnosis of important differentials. However, the counter argument is that the requesting cardiologists are seeking to investigate $C A D$ as the underlying cause of the chest pain and would defer to imaging stress tests as the alternative to CTCA, which would not detect extra-cardiac findings.

\section{Limitations}

Our data need to be interpreted in the context of the fact that although we have implemented the new NICE 2016 guidelines, a previous published audit of our RACPC demonstrated that we sent $9.4 \%$ of RACPC patients directly to ICA as they were deemed to be a high risk. A further $13.7 \%$ were referred to DSE as they were deemed not suitable for CTCA on 64-slice CT (mostly patients with asthma, COPD and ectopics). ${ }^{18}$

\section{Conclusion}

Our findings demonstrate that CTCA is an effective first-line test for most patients with new onset stable chest pain, as per NICE guidelines, as the majority were found to have normal/minimal disease. In the patients that went on to have ICA, CTCA had a relatively high yield of detecting severe CAD as confirmed on ICA (63\%). This compares well with our previous data applying the NICE 2010 guidelines, which recommended ICA for all higher probability patients, wherein the yield of severe CAD was $30 \%$. However, there are notable numbers of downstream tests for both CAD and incidental findings and their associated costs. 


\section{References}

1 Benjamin EJ, Virani SS, Callaway CW et al. Heart disease and stroke statistics - 2018 update: A report from the American Heart Association. Circulation 2018;137:e67-492.

2 National Institute for Health and Care Excellence. Chest pain of recent onset: assessment and diagnosis. Clinical guideline [CG95]. NICE, 2010.

3 Rauf A, Denny S, Pierres F et al. Current diagnostic yield of invasive coronary angiography at a district general hospital. $\mathrm{Br}]$ Cardiol 2017;24.

4 Cheng VY, Berman DS, Rozanski A et al. Performance of the traditional age, sex, and angina typicality-based approach for estimating pretest probability of angiographically significant coronary artery disease in patients undergoing coronary computed tomographic angiography: results from the multinational coronary CT angiography evaluation for clinical outcomes: an international multicenter registry (CONFIRM). Circulation 2011;124:2423-32, 1-8.

5 Patel MR, Peterson ED, Dai D et al. Low diagnostic yield of elective coronary angiography. N Engl J Med 2010;362:886-95.

6 National Institute for Health and Care Excellence. Chest pain of recent onset: Assessment and diagnosis of recent onset chest pain or discomfort of suspected cardiac origin. NICE guideline [CG95]. NICE, 2016.

7 Budoff MJ, Dowe D, Jollis JG et al. Diagnostic performance of 64-multidetector row coronary computed tomographic angiography for evaluation of coronary artery stenosis in individuals without known coronary artery disease: results from the prospective multicenter ACCURACY (Assessment by Coronary Computed Tomographic Angiography of Individuals Undergoing Invasive Coronary Angiography) trial. J Am Coll Cardiol 2008;52:1724-32.

8 Douglas PS, Hoffmann U, Patel MR et al. Outcomes of anatomical versus functional testing for coronary artery disease. N Engl J Med 2015;372:1291-1300.

9 Meijboom WB, Meijs MF, Schuijf JD et al. Diagnostic accuracy of 64-slice computed tomography coronary angiography: a prospective, multicenter, multivendor study. J Am Coll Cardiol 2008;52:2135-44.
10 Cremer PC, Nissen SE. The National Institute for Health and Care Excellence update for stable chest pain: poorly reasoned and risky for patients. Heart 2017;103:972-4.

11 Danad I, Raijmakers PG, Driessen RS et al. Comparison of coronary CT angiography, SPECT, PET, and hybrid imaging for diagnosis of ischemic heart disease determined by fractional flow reserve. JAMA Cardiol 2017;2:1100-7.

12 National Institute for Health and Care Excellence. HeartFlow FFRCT for estimating fractional flow reserve from coronary CT angiography. Medical technologies guidance [MTG32]. NICE, 2017.

13 Fyyaz S PA, Byrne J, Alfakih K. Opinions on the expanding role of CTCA in patients with stable chest pain and beyond: a UK survey. Br J Cardiol 2018;25:107-9.

14 Siontis GC, Mavridis D, Greenwood JP et al. Outcomes of non-invasive diagnostic modalities for the detection of coronary artery disease: network meta-analysis of diagnostic randomised controlled trials. BMJ 2018;360:k504.

15 The SCOT-HEART Investigators. Coronary CT Angiography and 5-Year Risk of Myocardial Infarction. N Engl J Med 2018;379:924-33.

16 Genders TS, Steyerberg EW, Alkadhi H et al. A clinical prediction rule for the diagnosis of coronary artery disease: validation, updating, and extension. Eur Heart J 2011;32:1316-30.

17 Robertson J, Nicholls S, Bardin P et al. Incidental pulmonary nodules are common on CT coronary angiogram and have a significant cost impact. Heart Lung Circ 2019;28:295-301.

18 Denny S, Vaughan G, Byrne J, Monaghan M, Alfakih K. The UK NICE guidelines on stable chest pain 2016: CT coronary angiography as the first line test for all patients presenting with stable chest pain. Open Heart (Unpublished data).

Address for correspondence: Dr Khaled Alfakih, University Hospital Lewisham, Lewisham High Street, London SE13 6LH, UK. Email:khaled.alfakih@nhs.net 